\title{
A new Mittag-Leffler function undetermined coefficient method and its applications to fractional homogeneous partial differential equations
}

\author{
YanQin Liu,a,b, HongGuang Sun ${ }^{a}$, XiuLing Yin ${ }^{b}$, BaoGui Xin \\ ${ }^{a}$ Institute of Soft Matter Mechanics, Department of Engineering Mechanics, Hohai University, Jiangsu 210098, China. \\ ${ }^{b}$ School of Mathematical Sciences, Dezhou University, Dezhou 253023, China. \\ ${ }^{c}$ Nonlinear Science Center, College of Economics and Management, Shandong University of Science and Technology, Qingdao \\ 266590, China.
}

Communicated by D. Baleanu

\begin{abstract}
In this paper, we develop a new application of the Mittag-Leffler function that will extend the application to fractional homogeneous differential equations, and propose a Mittag-Leffler function undetermined coefficient method. A new solution is constructed in power series. When a very simple ordinary differential equation is satisfied, no matter the original equation is linear or nonlinear, the method is valid, then combine the alike terms, compare the coefficient with identical powers, and the undetermined coefficient will be obtained. The fractional derivatives are described in the Caputo sense. To illustrate the reliability of the method, some examples are provided, and the solutions are in the form of generalized Mittag-Leffler function. The results reveal that the approach introduced here are very effective and convenient for solving homogeneous differential equations with fractional order. (c)2017 All rights reserved.
\end{abstract}

Keywords: Mittag-Leffler function, undetermined coefficient method, fractional homogeneous equation, Caputo derivative. 2010 MSC: $35 \mathrm{C} 05,35 \mathrm{C} 10$.

\section{Introduction}

Many phenomena in the physical, chemical, and biological sciences as well as in technologies are governed by differential equations. In recent years, fractional differential equations $[8,14,17]$ have attracted much attention in a variety of applied sciences, such as visco-elasticity, feed back amplifiers, electrical circuits, electro-analytical chemistry, fractional multiples [8, 10, 17], etc.. Consider the general fractional homogeneous partial differential equation

$$
D_{t}^{\alpha} u(x, t)=\sum_{i=1}^{n} a_{i} x D_{x_{i}}^{\delta_{i}} u(x, t)+A(u(x, t)),
$$

\footnotetext{
*Corresponding author

Email addresses: yqliumath@163.com (YanQin Liu), shg@hhu.edu.cn (HongGuang Sun), yinxiuling@dzu.edu.cn (XiuLing Yin), xin@tju.edu.cn (BaoGui Xin)

doi:10.22436/jnsa.010.08.43
} 
subject to the initial condition

$$
\frac{\partial^{j} u(x, 0)}{\partial t^{j}}=\varphi_{j}(x)
$$

where $m-1<\alpha \leqslant m, j=0,1,2, \cdots, m-1, m \in N, \delta_{i} \in N, 0 \leqslant t \leqslant T, x=\left(x_{1}, x_{2}, \cdots, x_{n}\right) \in R^{n}, a_{i}(x), \varphi_{j}(x)$ are functions of variable $x$, and $A(u(x, t))$ is linear or nonlinear in $u(x, t)$ and $u_{x}(x, t)$. Note that $D_{t}^{\alpha} u(x, t)$ is the $\alpha^{\text {th }}$-order Caputo partial fractional derivative of function $u(x, t)$ with respect to " $t$ ".

Note that equation (1.1) can be used to describe transport, diffusion-wave phenomena in some fields. And the importance of obtaining the exact and approximate solutions of fractional equations in physics and mathematics is still a significant problem that needs new methods to discover exact and approximate solutions. But these fractional differential equations are difficult to get their exact solutions [9, 12, 13, 21]. So, these types of equations are solved by various methods such as Adomian decomposition method $[1,22]$, variational iteration method $[7,20]$, differential transform method $[2,6]$, homotopy perturbation method [11, 15], an iterative method [4, 23], finite element method [19, 25], finite difference method [3, 18], etc..

We need to recall some definitions that will be used in the theory of fractional differential equations $[8,10,17]$.

Definition 1.1. The Gamma function is defined as follows:

$$
\Gamma(x):=\int_{0}^{\infty} t^{x-1} e^{-t} d t,(\operatorname{Re}(x)>0) .
$$

Definition 1.2. The Mittag-Leffler (1902-1905) function $E_{\alpha}$, is defined by the power series

$$
\mathrm{E}_{\alpha}(\mathrm{t}):=\sum_{\mathrm{k}=0}^{\infty} \frac{\mathrm{t}^{\mathrm{k}}}{\Gamma(\alpha \mathrm{k}+1)}, \alpha>0 .
$$

Definition 1.3. The Riemann-Liouville fractional integral of order $\alpha(\alpha>0)$ of a function $u(x, t)$ is denoted by $I_{t}^{\alpha} \mathfrak{u}(x, t)$ and defined as

$$
I_{t}^{\alpha} u(x, t):=\frac{1}{\Gamma(\alpha)} \int_{0}^{t}(t-\tau)^{\alpha-1} u(x, \tau) d \tau, t>0 .
$$

Definition 1.4. The Caputo partial fractional derivative of order $\alpha(\alpha>0)$ of a function $u(x, t)$ is denoted by $D_{t}^{\alpha} u(x, t)$ and defined as

$$
D_{t}^{\alpha} u(x, t):= \begin{cases}\frac{\partial^{m}}{\partial t^{m}} u(x, t), & \alpha=m, m \in N, \\ I_{t}^{m}-\alpha \frac{\partial^{m}}{\partial t^{m}} u(x, t), & m-1<\alpha<m, m \in N .\end{cases}
$$

Using Definition 1.4, it is obvious that when $\gamma>-1$ and $C \in R$, we have $D_{t}^{\alpha} t^{\gamma}=\frac{\Gamma(\gamma+1)}{\Gamma(\gamma+1-\alpha)} t^{\gamma-\alpha}$, $\mathrm{D}_{\mathrm{t}}^{\alpha} \mathrm{C}=0$, and

$$
D_{t}^{\alpha}\left(E_{\alpha}(t)\right)=D_{t}^{\alpha}\left(\sum_{k=0}^{\infty} \frac{t^{k}}{\Gamma(\alpha k+1)}\right)=\sum_{k=1}^{\infty} \frac{t^{k-1}}{\Gamma(\alpha(k-1)+1)}=\sum_{k=0}^{\infty} \frac{t^{k}}{\Gamma(\alpha k+1)}=E_{\alpha}(t) .
$$

\section{Description of the new Mittag-Leffler function undetermined coefficient method}

Now we discuss this new method, which is called Mittag-Leffler function undetermined coefficient method. To illustrate the basic idea of this method, take a simple fractional homogeneous equation as an example:

$$
D_{t}^{\alpha} u(x, t)=L(u(x, t))
$$


with the initial condition

$$
u(x, 0)=\varphi(x),
$$

where $D_{t}^{\alpha}$ is the $\alpha^{\text {th }}$-order Caputo partial fractional derivative, $0<\alpha \leqslant 1, \varphi(x)$ is a known function, and $L$ is a linear operator from a Banach space B to itself. In this paper, we will explain how to solve some of differential equations with fractional level through the imposition the generalized Mittag-Leffler function $E_{\alpha}(t)$, this function has already proved its efficiency as solutions of fractional calculus theory and applications, and its convergence was also discussed in [17]. The new Mittag-Leffler function undetermined coefficient method suggests that the solution $u(x, t)$ can be decomposed by an infinite series of components for some equations:

$$
u(x, t)=f(x) E_{\alpha}\left(A t^{\alpha}\right)=\sum_{n=0}^{\infty} f(x) A^{n} \frac{t^{n \alpha}}{\Gamma(n \alpha+1)},
$$

where $A$ is an undetermined coefficient, using the initial condition $u(x, 0)=\varphi(x)$, we have $f(x)=\varphi(x)$. substituting Eq. (2.2) into (2.1) and using the property, we get

$$
\sum_{n=1}^{\infty} \varphi(x) A^{n} \frac{t^{(n-1) \alpha}}{\Gamma((n-1) \alpha+1)}=L\left(\sum_{n=0}^{\infty} \varphi(x) A^{n} \frac{t^{n \alpha}}{\Gamma(n \alpha+1)}\right) .
$$

Combining the alike terms and replacing $n$ by $n+1$ in the first sum, we assume the form

$$
\sum_{n=0}^{\infty} \varphi(x) A^{n+1} \frac{t^{n \alpha}}{\Gamma(n \alpha+1)}=L(\varphi(x)) \sum_{n=0}^{\infty} A^{n} \frac{t^{n \alpha}}{\Gamma(n \alpha+1)} .
$$

If the equation has a solution of generalized Mittag-Leffler function, it must satisfy the following condition which is an ordinary differential equation

$$
\mathrm{L}(\varphi(x))=\mathrm{B} \varphi(x),
$$

and $B$ is a constant, then Eq. (2.3) turns into the following form

$$
\varphi(x) \sum_{n=0}^{\infty}\left(A^{n+1}-B A^{n}\right) \frac{t^{n \alpha}}{\Gamma(n \alpha+1)}=0,
$$

with the coefficient of $\mathrm{t}^{\mathrm{n} \alpha}$ equal to zero and identifying the coefficients, we obtain recursive

$$
\left\{\begin{array}{l}
A^{n+1}-B A^{n}=0 \Rightarrow A^{n+1}=B A^{n}, \\
\text { at } n=0, A^{1}=B A^{0}=B, \\
\text { at } n=1, A^{2}=B A^{1}=B^{2}, \\
\text { at } n=2, A^{3}=B A^{2}=B^{3},
\end{array}\right.
$$

so the undetermined coefficient $A$ is obtained, then substituting into (2.2), the general solution is

$$
u(x, t)=\varphi(x) E_{\alpha}\left(B t^{\alpha}\right) .
$$

Remark 2.1. For the fractional nonlinear equations, nonlinear term $N(u(x, t))$ can be decomposed as follows:

$$
\begin{aligned}
\mathrm{N}(\mathrm{u}(\mathrm{x}, \mathrm{t})) & =\mathrm{N}\left(\sum_{n=0}^{\infty} \varphi(x) A^{n} \frac{t^{n \alpha}}{\Gamma(n \alpha+1)}\right) \\
& =\mathrm{N}\left(\sum_{n=0}^{\infty} \varphi(x) u_{n}(t)\right)=\mathrm{N}(\varphi(x)) \mathrm{N}\left(\sum_{n=0}^{\infty} u_{n}(t)\right)=\mathrm{N}(\varphi(x))\left(\mathrm{N}\left(\mathrm{u}_{0}\right)+\sum_{n=1}^{\infty}\left(\mathrm{N}\left(\sum_{j=0}^{n} u_{j}\right)-N\left(\sum_{j=0}^{n-1} u_{j}\right)\right)\right) .
\end{aligned}
$$

If the equation has a solution of generalized Mittag-Leffler function, it must satisfy $N(\varphi(x))=0$ or $\mathrm{N}(\varphi(x))=\mathrm{C} \varphi(x)$, and $\mathrm{C}$ is a constant. 
Remark 2.2. For the fractional equation with derivative order $1<\alpha \leqslant 2$, and subject to the initial conditions $u(x, 0)=\varphi(x), \mathfrak{u}_{\mathfrak{t}}(x, 0)=\phi(x)$, if its solution $\mathfrak{u}(x, t)$ is also decomposed by an infinite series of components $\sum_{n=0}^{\infty} f(x) A^{n} \frac{t^{n \alpha}}{\Gamma(n \alpha+1)}$, combined with the initial conditions, we have $f(x)=\varphi(x)$ and $\phi(x)$ must be 0 , then repeating the above process, the undetermined coefficient will be gotten. If $\phi(x) \neq 0$, this Mittag-Leffler function undetermined coefficient method will be invalid, we must explore other effective methods.

\section{Applications and results}

In order to assess the advantages and the accuracy of the Mittag-Leffler function undetermined coefficient method, in this section, we discuss some illustrative examples for time fractional homogeneous equations.

Example 3.1. Consider the following one-dimensional linear time fractional homogeneous heat-like equation:

$$
D_{t}^{\alpha} u(x, t)=\frac{1}{2} \chi^{2} D_{x}^{2} u
$$

with boundary conditions

$$
u(0, t)=0, u(1, t)=e^{t}
$$

and the initial condition

$$
u(x, 0)=x^{2},
$$

where $0<\alpha \leqslant 1,0<x<1, t>0$. Denoting $L(u(x, t))=\frac{1}{2} x^{2} u_{x x}$, and

$$
\mathrm{L}\left(\chi^{2}\right)=\frac{1}{2} \chi^{2} \mathrm{D}_{x}^{2}\left(x^{2}\right)=\chi^{2}=\mathrm{u}(x, 0),
$$

this equation satisfies the condition (2.4), and $B=1$, so the new method is applicable, and the solution of this equation can be expressed in the generalized Mittag-Leffler function $u(x, t)=\sum_{n=0}^{\infty} x^{2} A^{n} \frac{t^{n \alpha}}{\Gamma(n \alpha+1)}$, substituting into Eq. (3.1), we find

$$
\sum_{n=1}^{\infty} x^{2} A^{n} \frac{t^{(n-1) \alpha}}{\Gamma((n-1) \alpha+1)}=x^{2} \sum_{n=0}^{\infty} A^{n} \frac{t^{n \alpha}}{\Gamma(n \alpha+1)}
$$

combining the alike terms and replacing $n$ by $n+1$ in the first sum

$$
\left\{\begin{array}{l}
\sum_{n=0}^{\infty} x^{2} A^{n+1} \frac{t^{n \alpha}}{\Gamma(n \alpha+1)}=x^{2} \sum_{n=0}^{\infty} A^{n} \frac{t^{n \alpha}}{\Gamma(n \alpha+1)} \\
x^{2} \sum_{n=0}^{\infty}\left(A^{n+1}-A^{n}\right) \frac{t^{n \alpha}}{\Gamma(n \alpha+1)}=0
\end{array}\right.
$$

With the coefficient of $t^{n \alpha}$ equal to zero and identifying the coefficients, we obtain

$$
A^{n+1}=A^{n}
$$

so $A=1$ and the solution of Eq. (3.1)-(3.2)-(3.3) is

$$
u(x, t)=x^{2} E_{\alpha}\left(t^{\alpha}\right) .
$$

Remark 3.2. Particularly, if $\alpha=1$, then the one-dimensional linear time fractional heat-like equation is the regular heat-like equation, and the exact solution is $x^{2} e^{t}$, which is consistent with the solution in [16]. 
Example 3.3. Consider the time fractional homogeneous diffusion equation:

$$
D_{t}^{\alpha} u(x, t)=D_{x_{1}}^{2} u(x, t)+D_{x_{2}}^{2} u(x, t)-u(x, t),
$$

with initial condition

$$
u(x, 0)=\sin x_{1} \sin x_{2}+\cos x_{1} \cos x_{2},
$$

where $0<\alpha \leqslant 1, x=\left(x_{1}, x_{2}\right) \in R^{2}, t>0$. Denoting $L(u(x, t))=D_{x_{1}}^{2} u(x, t)+D_{x_{2}}^{2} u(x, t)-u(x, t)$, and

$$
\begin{aligned}
\mathrm{L}\left(\sin x_{1} \sin x_{2}+\cos x_{1} \cos x_{2}\right)= & \mathrm{D}_{x_{1}}^{2}\left(\sin x_{1} \sin x_{2}+\cos x_{1} \cos x_{2}\right) \\
& +D_{x_{2}}^{2}\left(\sin x_{1} \sin x_{2}+\cos x_{1} \cos x_{2}\right)-\left(\sin x_{1} \sin x_{2}+\cos x_{1} \cos x_{2}\right) \\
= & -3\left(\sin x_{1} \sin x_{2}+\cos x_{1} \cos x_{2}\right)=-3 u(x, 0),
\end{aligned}
$$

this equation satisfies the condition (2.4), and $B=-3$, so the new method is applicable, and the solution of this equation can be expressed in the generalized Mittag-Leffler function $u(x, t)=\sum_{n=0}^{\infty}\left(\sin x_{1} \sin x_{2}+\right.$ $\left.\cos x_{1} \cos x_{2}\right) A^{n} \frac{t^{n \alpha}}{\Gamma(n \alpha+1)}$, substituting into Eq. (3.4), and combining the alike terms and replacing $n$ by $\mathrm{n}+1$ in the first sum, we find

$$
\sum_{n=0}^{\infty}\left(\sin x_{1} \sin x_{2}+\cos x_{1} \cos x_{2}\right) A^{n+1} \frac{t^{n \alpha}}{\Gamma(n \alpha+1)}=\sum_{n=0}^{\infty}\left(\sin x_{1} \sin x_{2}+\cos x_{1} \cos x_{2}\right)\left(-3 A^{n}\right) \frac{t^{n \alpha}}{\Gamma(n \alpha+1)},
$$

with the coefficient of $t^{\text {n } \alpha}$ equal to zero and identifying the coefficients, we obtain

$$
A^{n+1}=-3 A^{n},
$$

so $A=-3$ and the solution of Eq. (3.4)-(3.5) is

$$
u(x, t)=\left(\sin x_{1} \sin x_{2}+\cos x_{1} \cos x_{2}\right) E_{\alpha}\left(-3 t^{\alpha}\right) .
$$

Remark 3.4. The solution (3.6) is consistent with the result in [4]. Particularly, if $\alpha=1$, then the time fractional homogeneous diffusion equation is the regular diffusion equation, and the exact solution is $\left(\sin x_{1} \sin x_{2}+\cos x_{1} \cos x_{2}\right) e^{-3 t}$.

Example 3.5. Consider the fourth order time fractional homogeneous parabolic equation:

$$
D_{t}^{\alpha} u(x, t)=D_{x}^{4} u(x, t)+D_{x}^{2} u(x, t)+u(x, t),
$$

with initial condition

$$
u(x, 0)=\cosh x,
$$

where $0<\alpha \leqslant 1, x \in R, t>0$. Denoting $L(u(x, t))=D_{x}^{4} u(x, t)+D_{x}^{2} u(x, t)+u(x, t)$, and

$$
\mathrm{L}(\cosh x)=\mathrm{D}_{x}^{4}(\cosh x)+\mathrm{D}_{x}^{2}(\cosh x)+\cosh x=3 \cosh x=3 \mathrm{u}(x, 0),
$$

this equation satisfies the condition (2.4), and $B=3$, so the new method is applicable, and the solution of this equation can be expressed in the generalized Mittag-Leffler function $u(x, t)=\sum_{n=0}^{\infty} \cosh x A^{n} \frac{t^{n \alpha}}{\Gamma(n \alpha+1)}$, repeating the above process, we have $A=B=3$, so the solution of Eq. (3.7)-(3.8) is

$$
u(x, t)=\cosh x E_{\alpha}\left(3 t^{\alpha}\right) .
$$

Remark 3.6. The solution (3.9) is consistent with the result in [4]. Particularly, if $\alpha=1$, then the fourth order time fractional homogeneous parabolic equation is the regular parabolic equation, and the exact solution is $\cosh x e^{3 t}$. 
Example 3.7. In this case, we consider the time fractional homogeneous backward Kolmogorov equation:

$$
D_{t}^{\alpha} u(x, t)=(x+1) D_{x} u(x, t)+\left(x^{2} e^{t}\right) D_{x}^{2} u(x, t),
$$

with initial condition

$$
u(x, 0)=x+1
$$

where $0<\alpha \leqslant 1, x=\left(x_{1}, x_{2}\right) \in R^{2}, t>0$. Denoting $L(u(x, t))=(x+1) D_{x} u(x, t)+\left(x^{2} e^{t}\right) D_{x}^{2} u(x, t)$, and

$$
\mathrm{L}(x+1)=(x+1) \mathrm{D}_{x}(x+1)+\left(x^{2} e^{t}\right) D_{x}^{2}(x+1)=x+1+0=u(x, 0),
$$

this equation satisfies the condition (2.4), and $B=1$, so the new method is applicable, and the solution of this equation can be expressed in the generalized Mittag-Leffler function $u(x, t)=\sum_{n=0}^{\infty}(x+$ 1) $A^{n} \frac{t^{n \alpha}}{\Gamma(n \alpha+1)}$, substituting into Eq. (3.10), and combining the alike terms and replacing $n$ by $n+1$ in the first sum, we find

$$
\sum_{n=0}^{\infty}(x+1) A^{n+1} \frac{t^{n \alpha}}{\Gamma(n \alpha+1)}=\sum_{n=0}^{\infty}(x+1) A^{n} \frac{t^{n \alpha}}{\Gamma(n \alpha+1)}+0,
$$

so the solution of Eq. (3.10)-(3.11) is

$$
u(x, t)=(x+1) E_{\alpha}\left(t^{\alpha}\right) .
$$

Remark 3.8. The solution (3.12) is consistent with the result in [24]. Particularly, if $\alpha=1$, then the time fractional homogeneous backward Kolmogorov equation is the regular backward Kolmogorov equation, and the exact solution is $(x+1) e^{t}$.

Example 3.9. In this case, we consider the time fractional nonlinear homogeneous forward Kolmogorov equation:

$$
D_{t}^{\alpha} u(x, t)=-D_{x}\left(\frac{4 u}{x}-\frac{x}{3}\right) u+D_{x}^{2} u^{2}
$$

with initial condition

$$
u(x, 0)=x^{2},
$$

where $0<\alpha \leqslant 1, x \in R, t>0$. In the right side of the equation,

$$
-D_{x}\left(\frac{4 u}{x}-\frac{x}{3}\right) u+D_{x}^{2} u^{2}=D_{x}\left(\frac{x}{3} u\right)+D_{x}^{2}\left(u^{2}\right)-D_{x}\left(\frac{4 u^{2}}{x}\right)=L(u(x, t)+N(u(x, t))),
$$

where $L(u(x, t))=D_{x}\left(\frac{x}{3} u\right), N(u(x, t))=D_{x}^{2}\left(u^{2}\right)-D_{x}\left(\frac{4 u^{2}}{x}\right)$, and we check that

$$
\mathrm{L}\left(\mathrm{x}^{2}\right)=\mathrm{D}_{x}\left(\frac{x}{3} x^{2}\right)=x^{2}, \quad \mathrm{~N}\left(x^{2}\right)=\mathrm{D}_{x}^{2}\left(x^{2}\right)^{2}-\mathrm{D}_{x}\left(\frac{4\left(x^{2}\right)^{2}}{x}\right)=0,
$$

this equation satisfies the condition (2.4), and $B=1$, so the new method is applicable, and the solution of Eq. (3.13)-(3.14) is

$$
u(x, t)=x^{2} E_{\alpha}\left(t^{\alpha}\right) .
$$

Remark 3.10. The solution (3.15) is consistent with the result in [24]. Particularly, if $\alpha=1$, then the time fractional nonlinear homogeneous forward Kolmogorov equation is the regular forward Kolmogorov equation, and the exact solution is $x^{2} e^{t}$.

Example 3.11. Consider the time fractional homogeneous biological population equation which satisfies the Malthusian Law:

$$
D_{t}^{\alpha} u(x, y, t)=D_{x}^{2} u^{2}+D_{y}^{2} u^{2}+h u(x, y, t)
$$

with initial condition

$$
u(x, 0)=\sqrt{x y}
$$


where $0<\alpha \leqslant 1, x \in R, t>0, h$ is a constant. Denoting

$$
L(u(x, t)=) h u(x, y, t), N(u(x, t))=D_{x}^{2} u^{2}+D_{y}^{2} u^{2},
$$

and we check that

$$
\mathrm{L}(\sqrt{x y})=\mathrm{h} \sqrt{x y}, \quad \mathrm{~N}(\sqrt{x y})=0,
$$

this equation satisfies the condition (2.4), and $B=h$, so the new method is applicable, and the solution of Eq. (3.16)-(3.17) is

$$
u(x, t)=\sqrt{x y} E_{\alpha}\left(h t^{\alpha}\right) .
$$

Remark 3.12. The solution (3.18) is consistent with the result in [5]. Particularly, if $\alpha=1$, then the time fractional homogeneous biological population equation is the regular biological population equation, and the exact solution is $\sqrt{x y} e^{\text {ht }}$.

Example 3.13. Consider the time fractional homogeneous biological population equation which satisfies the Verhulst Law:

$$
D_{t}^{\alpha} u(x, y, t)=D_{x}^{2} u^{2}+D_{y}^{2} u^{2}+h u(1-r u),
$$

with initial condition

$$
u(x, 0)=e^{\frac{\sqrt{h r}}{8}(x+y)},
$$

where $0<\alpha \leqslant 1, x \in R, t>0, h, r$ are constant. Denoting

$$
\mathrm{L}(\mathrm{u}(\mathrm{x}, \mathrm{t}))=h \mathrm{u}, \mathrm{N}(\mathrm{u}(\mathrm{x}, \mathrm{t}))=\mathrm{D}_{x}^{2} \mathrm{u}^{2}+\mathrm{D}_{y}^{2} \mathrm{u}^{2}-\mathrm{hru} \mathrm{u}^{2},
$$

and checking that

$$
L\left(e^{\frac{\sqrt{h r}}{8}(x+y)}\right)=h e^{\sqrt{h r} 8(x+y)}, \quad N\left(e^{\frac{\sqrt{h r}}{8}(x+y)}\right)=0,
$$

this equation satisfies the condition (2.4), and $B=h$, so the new method is applicable, and the solution of Eq. (3.19)-(3.20) is

$$
u(x, t)=e^{\frac{\sqrt{h r}}{8}(x+y)} E_{\alpha}\left(h t^{\alpha}\right) .
$$

Remark 3.14. The solution (3.21) is consistent with the result in [5]. Particularly, if $\alpha=1$, then the time fractional homogeneous biological population equation is the regular biological population equation, and the exact solution is $e^{\frac{\sqrt{h r}}{8}(x+y)} e^{\text {ht }}$.

Example 3.15. Consider the general fourth-order time fractional Boussinesq equation with one-dimensional space variable:

$$
D_{t}^{\alpha} \mathfrak{u}(x, t)=\beta D_{x}^{4} \mathfrak{u}(x, t)+\gamma D_{x}^{2} \mathfrak{u}(x, t)+\theta D_{x}^{2} u^{2}(x, t)-4 \theta u^{2}(x, t),
$$

with initial condition

$$
u(x, 0)=e^{x}, \quad u_{t}(x, 0)=0,
$$

where $1<\alpha \leqslant 2, x \in R, t>0, \beta, \gamma$ and $\theta$ are constant coefficients. Denoting

$$
\mathrm{L}(\mathrm{u}(x, \mathrm{t}))=\beta \mathrm{D}_{\chi}^{4} \mathfrak{u}(x, \mathrm{t})+\gamma \mathrm{D}_{x}^{2} \mathrm{u}(x, \mathrm{t}), \mathrm{N}(\mathrm{u}(x, \mathrm{t}))=\theta \mathrm{D}_{\chi}^{2} \mathrm{u}^{2}(x, \mathrm{t})-4 \theta \mathrm{u}^{2}(x, \mathrm{t}),
$$

and checking that

$$
\mathrm{L}\left(e^{\mathrm{x}}\right)=(\beta+\gamma) e^{\mathrm{x}}, \quad \mathrm{N}\left(\mathrm{e}^{\mathrm{x}}\right)=0,
$$

this equation satisfies the condition (2.4), and $B=\beta+\gamma$, so the new method is applicable, and the solution of Eq. (3.22)-(3.23) is

$$
u(x, t)=e^{x} E_{\alpha}\left((\beta+\gamma) t^{\alpha}\right) .
$$


Remark 3.16. The solution (3.24) is consistent with the result in [23]. Particularly, if $\alpha=2$, then the general fourth-order time fractional Boussinesq equation with one-dimensional space variable is the regular fourth-order Boussinesq equation, and the exact solution is $e^{x} E_{2}\left((\beta+\gamma) t^{2}\right)$.

Example 3.17. Let us consider the sixth-order time fractional Boussinesq equation:

$$
D_{t}^{\alpha} u(x, t)=D_{x}^{6} u(x, t)+D_{x}^{4} u(x, t)+D_{x}^{2} u(x, t)+D_{x}^{2} u^{2}(x, t)-4 \theta u^{2}(x, t),
$$

with initial condition

$$
u(x, 0)=e^{x}, \quad u_{t}(x, 0)=0,
$$

where $1<\alpha \leqslant 2, x \in R, t>0$. Denoting

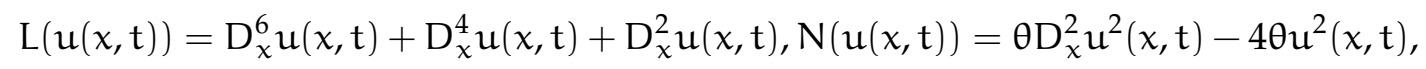

and checking that

$$
\mathrm{L}\left(e^{\mathrm{x}}\right)=3 e^{\mathrm{x}}, \quad \mathrm{N}\left(\mathrm{e}^{\mathrm{x}}\right)=0,
$$

this equation satisfies the condition (2.4), and $B=3$ so the new method is applicable, and the solution of Eq. (3.25)-(3.26) is

$$
u(x, t)=e^{x} E_{\alpha}\left(3 t^{\alpha}\right)
$$

Remark 3.18. The solution (3.27) is consistent with the result in [23]. Particularly, if $\alpha=2$, then sixth-order time fractional Boussinesq equation is the regular sixth-order Boussinesq equation, and the exact solution is $e^{x} E_{2}\left(3 t^{2}\right)$.

\section{Conclusion}

In this work, a new Mittag-Leffler function undetermined coefficient method is proposed and used to solve fractional homogeneous differential equations. This method is very simple, only need to verify weather an ordinary differential equation is right, when the ordinary differential equation is not satisfied, the method is invalid. By using this new method, we can get the analytical solution successfully. This method is effective and direct, which can be applied to other kinds of fractional homogeneous equations.

\section{Acknowledgment}

We express our thanks to the referees for their fruitful advices and comments. This work is supported by National Natural Science Foundation of China (11501082), Natural Science Foundation of Shandong Province (ZR2016AQ07) and Postdoctoral Science Foundation of Jiangsu Province (1402046C).

\section{References}

[1] G. Adomian, A review of the decomposition method in applied mathematics, J. Math. Anal. Appl., 135 (1988), 501-544. 1

[2] A. Al-rabtah, V. S. Ertürk, S. Momani, Solutions of a fractional oscillator by using differential tranform method, Comput. Math. Appl., 59 (2010), 1356-1362. 1

[3] S. Chen, F. Liu, I. Turner, V. Anh, An implicit numerical method for the two-dimensional fractional percolation equation, Appl. Math. Comput., 219 (2013), 4322-4331. 1

[4] C. D. Dhaigude, V. R. Nikam, Solutions of fractional partical differentical equations using iterative method, Fract. Calc. Appl. Anal., 15 (2012), 684-699. 1, 3.4, 3.6

[5] A. M. A. El-Sayed, S. Z. Rida, A. A. M. Arafa, Exact solutions of fractional-order biological population model, Commun. Theor. Phys., 52 (2009), 992-996. 3.12, 3.14

[6] V. S. Ertürk, S. Momani, Z. Odibat, Application of generalized differential transfom method to multi-order fractional differential equations, Commun. Nonlinear Sci., 13 (2008), 1642-1654. 1

[7] J.-H. He, Variational iteration method- a kind of nonlinear analytical technique: some examples, Int. J. Nonlinear Mech., 34 (1999), 699-708. 1 
[8] R. Hilfer, Applications of fractional calculus in physics, World scientific, Singapore, (2000). 1, 1

[9] X.-Y. Jiang, M.-Y. Xu, Analysis of fractional anomalous diffusion caused by an instantaneous point source in disordered fractal media, Int. J. Nonlinear Mech., 41 (2006), 156-165. 1

[10] A. A. Kilbas, H. M. Srivastava, J. J. Trujillo, Theory and applications of fractional differential equations, Elsevier, Amsterdam, (2006). 1, 1

[11] X.-C. Li, M.-Y. Xu, X.-Y. Jiang, Homotopy perturbation method to time-fractional diffusion equation with a moving boundary condition, Appl. Math. Comput., 208 (2009), 434-439. 1

[12] Y.-Q. Liu, J.-H. Ma, Exact solutions of a generalized multi-fractional nonlinear diffusion equation in radical symmetry, Commun. Theor. Phys., 52 (2009), 857-861. 1

[13] J.-H. Ma, Y.-Q. Liu, Exact solutions for a generalized nonlinear fractional Fokker-Planck equation, Nonlinear Anal. Real World Appl., 11 (2010), 515-521. 1

[14] R. Metzler, J. Klafter, The random walks guide to anomalous diffusion: a fractional dynamics approach, Phys. Rep., 339 (2000), 1-77. 1

[15] M. A. Noor, Some iterative methods for solving nonlinear equations using homotopy perturbation method, Int. J. Comput. Math., 87 (2010), 141-149. 1

[16] M. A. Noor, S. T. mohyud-Din, Modified variation iteration method for heat and wave-like equations, Acta Appl. Math., 104 (2008), 257-269. 3.2

[17] I. Podlubny, Fractional differential equations, Academic Press, New York, (1999). 1, 1, 2

[18] H.-G. Sun, W. Chen, C.-P. Li, Y.-Q. Chen, Finite difference schemes for variable-order time fractional diffusion equation, Internat. J. Bifur. Chaos Appl. Sci. Engrg., 2012 (2012), 16 pages. 1

[19] H.-G. Sun, W. Chen, K. Y. Sze, A semi-discrete finite element method for a class of time-fractional diffusion equatons, Phil. Trans. R. Soc. A, 2013 (2013), 15 pages. 1

[20] A.-M. Wazwaz, The variational iteration method for analytic treatment for linear and nonlinear ODEs, Appl. Math. Comput., 212 (2009), 120-134. 1

[21] S.-W. Wang, M.-Y. Xu, Axial couette flow of two kinds of fractional viscoelastic fluids in an annulus, Nonlinear Anal. Real World Appl., 10 (2009), 1087-1096. 1

[22] A.-M. Wazwaz, S. M. El-Sayed, A new modification of the Adomian decomposition method for linear and nonlinear operators, Appl. Math. Comput., 122 (2001), 393-405. 1

[23] F. Xu, Y.-X. Gao, W.-P. Zhang, Construction of analytic solution for time-fractional Boussinesq equation using iterative method, Adv. Math. Phys., 2015 (2015), 7 pages. 1, 3.16, 3.18

[24] A. Yildirim, Application of the homotopy perturbation method for the Fokker-Planck equation, Int. J. Numer. Methods Biomed. Eng., 26 (2010), 1144-1154. 3.8, 3.10

[25] P. Zhuang, F. Liu, I. Turner, Y. T. Gu, Finite volume and finite element methods for solving a one-dimensional spacefractional Boussinesq equation, Appl. Math. Model., 38 (2014), 3860-3870. 1 